\title{
Cell Metabolomics Responses to Chlorpromazine: Novel Insights Into Blood Brain Barrier Dysfunctionality
}

Surachai Ngamratanapaiboon ( $\square$ surachai.n@nmu.ac.th )

Navamindradhiraj University

\section{Research Article}

Keywords: Blood brain barrier, Dysfunction, Endothelial cells, Chlorpromazine, Cell metabolomics, Liquid chromatography mass spectrometry

Posted Date: August 10th, 2021

DOI: https://doi.org/10.21203/rs.3.rs-776559/v1

License: (a) (i) This work is licensed under a Creative Commons Attribution 4.0 International License.

Read Full License 


\section{Abstract}

Blood-brain barrier (BBB) prevents drug access and impedes therapeutic efficacy. Effective methods of modulating barrier function and resolving these difficulties are desperately needed. We hypothesized that cell metabolic adaptation significantly influences physiological and pathological barrier functionality because we were convinced that a better understanding of cell-oriented BBB responses could provide valuable insight, and because metabolic dysregulation is prominent in many vascular-related pathological processes associated with BBB disruption. Biochemical fingerprints of primary brain endothelial cells (EC) were obtained using untargeted liquid chromatography-mass spectrometry (LCMS) metabolomic profiling during chlorpromazine treatment, at concentration similar to its measured in the serum of high dosed patients, providing a functional readout of cell status. Metabolomic analyses showed brain EC had a distinct metabolic signature. Corroborating their role in BBB and CNS protection. Surprisingly, EC largely maintained their normoxic characteristics in drug treatment situations and their profiles diverged from those of control. When examining EC reactions, tissue specificity/origin is definitely significant. With a focus on cellular metabolism, we examine how cell metabolic adaptive capabilities may influence vascular stability, as well as the prospect that modifying metabolite levels may be an efficient strategy to modulate brain EC function. Overall, this study sheds new light on cell-associated metabolic changes and serves as a valuable resource for understanding BBB modifications in drug treatment circumstances.

\section{Introduction}

The cerebral vasculature regulates and limits biomolecule movement between blood and the CNS parenchyma via the blood-brain barrier (BBB) [1]. While specialized brain endothelial cells are physically connected via unique belt-like tight junctions that mediate BBB tightness, perivascular cells and astrocytes also play a role in BBB physiology, forming the neurovascular unit as a whole (NVU) [2]. It is evident that at chlorpromazine, at concentration similar to its measured in the serum of high dosed patients, is already established to induce gross permeability changes in the BBB [3, 4]. A few evident suggest that at high therapeutic concentration of chlorpromazine is likely to incur cytotoxic effects and apoptosis of BBB endothelial with an impairment of barrier functionality [4]; however, the cellular mechanism remain unclear.

Endothelial cells (EC) in the brain create the barrier, which is supported by perivascular cells such as astrocytes and pericytes, which release growth factors and substrates to maintain optimal barrier function $[5,6]$. Currently there still have a limited grasp of how these barrier-associated cells respond individually or collaborate - either healthily or pathologically. It is particularly unclear how alterations in cell-specific metabolism relate to barrier homeostasis. Better understanding could lead to more natural, and potentially very effective, strategies to regulate barrier properties [7].

To date, no detailed metabolomic profiling of brain microvascular ECs has been published. The brain endothelium is different in that it has a particular proteomic makeup and exhibits unanticipated 
molecular modifications upon damage, such as increased protein production, implying that specialized functions continue despite difficult conditions [8]. Furthermore, angiogenesis, the process that leads to increased vascular permeability, is primarily active in brain EC during protracted or severe damage situations $[9,10]$. These unique traits most likely amplify their crucial barrier role, implying that further research is needed to better understand their distinctive qualities. EC metabolism is tuned to constantly facilitate brain barrier function. Reduction of mitochondrial function and cellular bioenergetics enables brain EC to deal with high metabolic stress [11-13]. This ability to change metabolically quickly is critical for the surrounding neuron but whether similar or distinct metabolic coordination occurs in brain EC is unknown.

By studying BBB cell metabolomic alterations during chlorpromazine treatment, at concentration similar to its measured in the serum of high dosed patients, we hoped to gain a more complete understanding of brain EC responses. When researching the BBB in vivo, a lot of difficulties arise, but one of the most difficult is determining where specific signals originate and/or the impact of changing conditions on EC cell populations. We performed metabolomic characterization of primary brain microvascular EC to overcome these challenges. Untargeted metabolomics-based liquid chromatography-mass spectrometry (LC-MS) offered a comprehensive picture of cellular metabolite composition before and after exposure to drugs, imitating various clinical conditions. We show that brain EC profiles differ significantly when compared to control (untreated) and chlorpromazine-mediated changes in metabolite levels, and that brain EC profiles also differ from those of non-brain origin. We conclude that stressed EC are likely to be significantly reliant on drug treatment in cells after analyzing key metabolic pathways. Indeed, the observed EC after treatment suggests that it serves as a significant metabolic resource and underpins their potential to reduce EC function and integrity. Overall, this study sheds fresh light on cell metabolic changes and serves as a valuable resource for understanding BBB modifications throughout various drug treatment scenarios.

\section{Materials And Methods}

\section{Chemicals and reagents}

Mass spectrometer grade acetonitrile, chloroform, isopropanol, and methanol were purchased from APEX Chemical Co Ltd (Bangkok, Thailand), formic acid from DKSH Co Ltd (Bangkok, Thailand), and ammonium acetate from SMCHEM Co Ltd (Bangkok, Thailand). HPLC grade water was obtained from an ELGA system with $18.2 \mathrm{\Omega}$ (BEC Thail Chemical Equipment Co Ltd, Bangkok, Thailand). Chlorpromazine was purchased from Sigma-Aldrich Co Ltd (Bangkok, Thailand).

\section{Brain endothelia cell experiment}

Brain ECs were treated with DMSO as a control and $3 \mu \mathrm{M}$ chlorpromazine for $24 \mathrm{hrs}$. this concentration used in the current study is near to the top pf therapeutic serum levels and is similar to this measured as an overdosed in patients [12,13]. Each of the sample groups has six biological replicates. Briefly, brain ECs from human BBB were provided by Paul A. Smith (Faculty of Medicine \& Health Sciences, University 
of Nottingham Medical School, Queen's Medical Centre, Nottingham, NG7 2UH, UK) and used in passages 21-23 at density of $10^{8} \mathrm{cell} / \mathrm{ml}$. Brain ECs were cultured and maintained as described in Elmorsy et al. $[12,13]$. When $80 \%$ confluence was achieved, unattached brain ECs and cell culture media were discarded and attached cells were washed with $5 \mathrm{ml}$ of phosphate buffer solution. Next, $10 \mathrm{ml}$ of fresh cell culture media was added, and followed by $0.5 \mathrm{ml}$ of cold methanol $\left(4^{\circ} \mathrm{C}\right)$. Then a cell scraper was used to detached cell from a flask and the cell suspension was transferred to a cold Eppendorf tube. The suspension cell was then vigorously vortexed for $5 \mathrm{~min}$. After that $0.50 \mathrm{ml}$ of $4^{\circ} \mathrm{C}$ chloroform and $0.50 \mathrm{ml}$ of $4^{\circ} \mathrm{C}$ water were added to the suspension and then vortexed vigorously for 10 minutes at $4^{\circ} \mathrm{C}$. the suspension was centrifuged at $15,000 \mathrm{~g}$ for 10 minutes at $4^{\circ} \mathrm{C}$. The polar (water soluble) fraction and non-polar (lipid) fraction were collected separately and transferred into fresh Eppendorf tubes and evaporated to dryness at room temperature with $\mathrm{N}_{2}$ gas. The dried non-polar layer was reconstituted in 50 $\mu \mathrm{l}$ of chloroform and methanol $(1: 2 \mathrm{v} / \mathrm{v})$, and the dried polar layer was reconstituted in $50 \mu \mathrm{l}$ of water. The samples were centrifuged at $15,000 \mathrm{~g}$ for 10 minutes at $4^{\circ} \mathrm{C}$ to remove any cell debris and the solution was then transferred into a HPLC vial for LC-MS analysis.

\section{Liquid chromatography-mass spectrometry experimental}

Cellulat metabolite analysis was performed on an LC AccelaTM system (Thermo Scientific Ltd, Loughborough, UK) coupled with high resolution mass spectrometry (Exactive, Thermo Scientific Ltd, Loughborough, UK). Non-polar chromatographic separations were performed on an Agilent SB C8 column (1.8 $\mu \mathrm{m}$ particle size, $2.1 \times 100 \mathrm{~mm}$, Crawford Scientific Ltd., Lanarkshire, UK) and polar chromatographic separations were performed on a C18 (2) column $(2.5 \mu \mathrm{m}$ particle size, $3 \times 100 \mathrm{~mm}$, Phenomenex Ltd, Cheshire, UK). The details of LC and MS conditions are summarized in Table 1 and 2. In both non-polar and polar metabolic profiling, samples were performed in six replicates, to account for any biological variability. The retention time consistency and mass accuracy were confirmed though the pooled QC samples.

Principal component analysis (PCA) and orthogonal partial least squares discriminant analysis (OPLSDA) were employed to process the acquired LC-MS data. Samples were grouped together for OPLS-DA modelling. The PCA and OPLS-DA results were displayed as scores plots to visualize sample clustering and to indicate sample similarity. Discriminatory metabolites between the treated and the control for each antipsychotic drug were first screened with the variable importance in the projection (VIP) ranks $>1.00$ and then validated using ANOVA statistical analysis of false discovery rate (FDR) with a significant level of 0.05 for antipsychotic treatments. According to the identity check based on raw data and the features of peaks, the target masses of candidate metabolites identified in the profiling process were searched over a narrow $\pm 5 \mathrm{ppm}$ mass window in the HMDB, METLIN and LIPIDMAP databases. The possible pathway analysis combines results from the publications to help our studies identify the most relevant pathways involved in the conditions under study. Potential chlorpromazine effects on brain ECs were recognized based on the identified metabolic pathways and statistics. 


\section{Results}

\section{Data quality}

Figure 1 depicts LC-MS chromatograms for the non-polar fraction in ESI+ (a) and ESI-(b), and for the polar fraction in ESI+ (c) and ESI- (d). Throughout the analysis procedure, pooled QC (as shown in Figure 1 (e)(h)) samples were analyzed during analysis for both non-polar and polar fractions. During data collection, pooled QC samples were collected for analysis. The data from the pooled QC samples of both the nonpolar fraction and the polar fraction were then analyzed to identify the number of ions present, their intensity, and their percent RSD values.

The average RSDs of peak abundance for the pooled QC samples were 17.9 percent with a standard deviation of 7.84 percent for the lipid fraction and 22.86 percent with a standard deviation of 10.17 percent for the polar fraction. Because the FDA recommends that biochemical analysis ions have a percent RSD of less than 30 , these were employed in the subsequent examination of the test and control sample data. Our average percent RSD findings revealed that the system was extremely stable during the analysis procedure for both non-polar and polar fractions.

The aligned data array for both lipid fraction and polar fraction ion MS data were filtered using pooled non-polar and pooled polar QC samples for data analysis. In accordance with the FDA's

recommendations for biomarker analysis ions, those with a percent RSD of less than 30 were used in the subsequent study of brain ECs treated with chlorpromazine at high therapeutic dosage and control cell sample data. Consequently, the pooled QC filters were produced in such a way that ions were present in the pooled QC samples and had a variability of less than $30 \%$ RSD (abundance intensity). Profiling program generated a data array of 961 ions for the non-polar fraction and 860 mass ions for the polar fraction. This satisfied the above-mentioned conditions for each fraction.

\section{Effects of chlorpromazine on non-polar metabolites in brain endothelia cells}

PCA scores plots from our data revealed a strong distinction between control and chlorpromazine treatment of ECs in the non-polar fraction (Figure 2a in ESI+ and 2b) in ESI-. OPLS-DA (see Figure 2c in $\mathrm{ESI}+$ and 2d) in ESI- was then used to visualize samples in an attempt to differentiate between control and chlorpromazine treatment, revealing a very evident split in the non-polar fraction. VIP statistics (VIP > 1.0) were initially utilized to pre-select observed mass ions in order to discover which detected ions account for such a considerable separation in the case of OPLS-DA modeling. Then, from the observed mass ions with FDR (ANOVA) 0.05, the ones most connected with the OPLS-DA discriminant scores were chosen to reduce the danger of false positives in the selection of significantly altered mass ions. The 57 identified metabolites by exact mass from chlorpromazine treatment on brain ECs were found to be significantly different from the control using these criteria. These metabolites played the most important role in distinguishing the metabolic profiles of control and chlorpromazine group, as shown in Supplementary Table 1. Figure 3 shows the top ten significantly-identified changes in levels of detected metabolites. 
Interestingly, all of the top significant metabolites detected were found to be hydrophobic compounds. There were five lipid compounds (25-O-(2"-beta-D-glucopyranosyl-beta-D-glucopyranosyl)-25-hydroxy-11Eeicosenoic acid or 3-0-(alpha-L-rhamnopyranosyl-(1-2)-alpha-L-rhamnopyranosyl)-3-hydroxydecanoyl-3hydroxydecanoic acid, 2 diacylglycerols, 1-(9Z,12Z,15Z-octadecatrienoyl)-2-(11Z-eicosenoyl)-sn-glycerol, and linolenyl palmitate that were significantly changed by chlorpromazine treated the brain ECs. Butyl 4'O-butanoyl-6-0-hexadecanoyl-neohesperidoside, $\mathrm{N}$-(hexadecanoyl)-sphinganine-1-phosphate and lysophosphoethanolamine were found to be significantly increased in chlorpromazine-treated cells. In addition, D-pantethine was significantly changed, too.

\section{Effects of chlorpromazine on polar metabolites in brain endothelia cells}

Unsupervised PCA was used to separate the groups for global metabolic profiling. PCA score plots from our data did not demonstrate a clear distinction of control and chlorpromazine treatment in the polar fraction in both ESI+ and ESI- as shown in Figure 4a and 4b. The samples were then visualized using OPLS-DA in an attempt to differentiate between control and drug treatment, which demonstrated a very significant split in this fraction as shown in Figure $4 \mathrm{c}$ and $4 \mathrm{~d}$ in ESI+ and ESI-, respectively. The nineteen discovered metabolites from antipsychotic treatment on brain ECs were obtained using the criteria described in the previous section, as summarized in Supplementary Table 2. These metabolites were the most useful in distinguishing between the metabolic profiles of control and chlorpromazine treatment. Figure 5 provides the top ten significant level changes of the identified metabolites. Intriguingly, four of the top significant metabolites detected (1,2-dihexanoyl-sn-glycero-3-phosphate, 3-hydroxymelatonin, acetoacetic acid and butyrylcarnitine) were found to be significantly increased.

\section{Biological significant of the metabolic effects of chlorpromazine on brain endothelia cells}

Various changes in levels of non-polar compounds and polar metabolites were determined to characterize biological pathways that may be affected by chlorpromazine at high therapeutic dose in ECs and distinguish them from control cells. These are summarized in Supplementary Table 1 and 2.

Antipsychotic medication treatment resulted in changes in the levels of non-polar compounds and polar metabolites. In summary, as shown in Figure 6, the significantly altered non-polar compounds and polar metabolites were linked to bioenergetics and lipid metabolism.

\section{Discussion}

The goal of this study was to describe the results of an untargeted lipidomic and metabolomic analysis of the effects of chlorpromazine at high therapeutic doses in brain endothelial cells using cell-based LCMS metabolomics. This method has become increasingly popular and widely used in recent years [1416]. The analyses presented in this study addresses BBB dysfunction and breakdown caused by high antipsychotic therapeutic dosage.

\section{Chlorpromazine effects on brain endothelia cells}


Our findings show that brain ECs treated with chlorpromazine at high therapeutic doses have significantly altered levels of non-polar and polar metabolites (as summarized in Supplementary Table 1 and 2) when compared to untreated cells. These findings are consistent with, and significantly add to, previous reports of lipid metabolism and cellular metabolism changes in the BBB $[4,12]$. Taken together, the findings suggest that high therapeutic doses of antipsychotics may be involved in BBB dysfunction.

Interestingly, examining the results of non-polar and polar metabolite changes reveals that chlorpromazine at high therapeutic doses affect the lipid profiles and intracellular metabolic profiles of brain ECs. In cellular processes, lipid metabolism is mainly involved in mitochondrial functions $[17,18]$. As a result, changes in lipid profiles and metabolic profiles may impair mitochondrial function. Antipsychotics have been shown to impair mitochondrial function [19-21] as well as cellular and mitochondrial membrane structure [12,19,22], leading to cell apoptosis [23-25].

Mitochondria are intricate organelles found in all human cells. They are in charge of producing the vast majority of cellular energy in the form of adenosine triphosphate (ATP). ATP is the primary source of energy for almost all cellular processes. Impaired mitochondrial function leads to impaired bioenergetics, which results in a lack of cellular energy $[4,12,19]$. Elmorsy et al. $[4,12,22]$ recently demonstrated bioenergetic impairment in brain ECs treated with antipsychotics and subsequent mitochondrial dysfunction.

In brain ECs treated with chlorpromazine, the levels of acetoacetic acid, D-pantethine, (iso)butyrylcarnitine, 3-hydroxyactanoyl carnitine, L-carnitine, succinic acid semialdehyde, and D-arabitol significantly changed as summarized in Supplementary Table 1 and 2. As illustrated in Figure 6, changes in these metabolites may be involved in mitochondrial dysfunction. Mitochondrial dysfunction impairs bioenergetics in chlorpromazine-treated ECs. As a compensatory mechanism for energy deficiency, fatty acids are used for energy production. Several acid substances are produced during fatty acid metabolism, including acyl carnitine compounds and related compounds, as well as acetoacetic acid. The levels of acyl carnitine compounds ((iso)butyryl carnitine and 3-hydroxyactanoyl carnitine) in our results were significantly higher, while carnitine (an amino acid that plays an important role in fatty acid transport into mitochondria was significantly lower. These changes could confirm that brain ECs treated with high doses of chlorpromazine have a high rate of fatty acid metabolism.

Furthermore, the levels of acetoacetic acid and D-pantethine were found to be significantly higher. ECs and other cells do not normally contain high levels of acetoacetic acid and D-pantethine. These metabolites increase when cells lack oxaloacetic acid for the conversion of acetyl-CoA to citric acid or when fatty acid metabolism is excessive [26,27]. The levels of fatty acids and related compounds were significantly altered as a result of the findings. These changes could imply that the rise in acetoacetic acid and D-pantethine levels is due to mitochondrial dysfunction and an excess of fatty acid metabolism, which results in an excess of acetyl-CoA. Excess acetyl-CoA is then converted to acetoacetic acid. A state of ketoacidosis is reached when even more acetoacetic acid accumulates, lowering the cell's $\mathrm{pH}$ to 
dangerously acidic levels. Ketoacidosis is a metabolic disorder characterized by high levels of ketone bodies (acetoacetic acids), which causes EC dysfunction and apoptosis [28].

Furthermore, mitochondrial dysfunction and increased fatty acid metabolism can result in reactive oxygen species (ROS), which causes oxidative stress in cells. This stress can severely damage all cell components, including DNA, protein, and lipids [29]. Elmorsy et al. [12] demonstrated that antipsychotc treatment disrupted the cellular structure and morphology of brain ECs. The antipsychotics caused this disruption, which was linked to oxidative stress. Our results revealed pronounced changes in some lipid compounds in brain ECs treated with chlorpromazine. These lipids are cellular membrane constituents that play roles in cell proliferation, differentiation, apoptosis, and oxidative stress [30].

Moreover, there are a variety of antioxidant defenses in cells to detoxify fluctuations in ROS and its reactive intermediates, but ROS generation frequently exceeds the antioxidant capacity of cells [31]. The results show that the levels of hydroxymelatonin in brain ECs treated with chlorpromazine were significantly increased and decreased, respectively. Hydroxymelatonin is a byproduct of melatonin's interaction with reactive oxygen species (ROS) in cells [32]. Melatonin has been shown to act as a direct scavenger of ROS (thus not only regulating sleep and circadian rhythms) and to produce hydroxymelatonin in human cells [33]. The level of 3-hydroxymelatonin in cells is proportional to the level of oxidative stress caused by ROS [34]. Antipsychotics have been shown to increase the production of ROS in cells $[35,36]$. The findings, which are consistent with and significantly add to previous reports of ROS-induced oxidative stress in brain ECs, could imply that chlorpromazine is involved in ROS generation, resulting in oxidative stress.

In conclusion, chlorpromazine at high therapeutic dosage could affect the bioenergetics pathway due to mitochondrial dysfunction resulting in keto-acidosis and inducing oxide stress by reactive oxygen species generation in brain ECs.

\section{Declarations}

\section{Ethics approval and consent to participate}

Not applicable

\section{Consent for publication}

Not applicable

\section{Availability of data and material}

The datasets generated during and/or analyzed during the current study are available from the corresponding author on reasonable request.

\section{Competing interests}


Not applicable

\section{Funding}

This research did not receive any specific grant from funding agencies in the public, commercial, or notfor-profit sectors.

\section{Authors' contributions}

S.Ngamratanapaiboon wrote, prepared, and reviewed the manuscript.

\section{Acknowledgement}

I would like to thank Paul A. Smith (Faculty of Medicine \& Health Sciences, University of Nottingham Medical School, Queen's Medical Centre, Nottingham, NG7 2UH, UK) for cell culture experiments.

\section{Authors' information}

Surachai Ngamratanapaiboon earned Ph.D. degree in Pharmacy from the University of Nottingham, U.K., and Ph.D. in Biopharmaceutical Science from Mahidol University, Thailand. I am currently a staff in Division of Pharmacology, Department of Basic Medical Sciences, Faculty of Medicine Vajira Hospital, Navamindradhiraj University, Bangkok, 10300, Thailand. My current research focuses on pharmacology and toxicology studies based on targeted and untargeted metabolomics.

Tel: $+66-244-3000$ ext.3775

E-mail address: surachai.n@nmu.ac.th

\section{References}

1. Berghoff SA, Düking T, Spieth L, Winchenbach J, Stumpf SK, Gerndt N, et al (2017) Blood-brain barrier hyperpermeability precedes demyelination in the cuprizone model. Acta Neuropathol Commun [Internet]. 5(1):94. https://doi.org/10.1186/s40478-017-0497-6

2. Daneman R, Prat A (2015) The blood-brain barrier. Cold Spring Harb Perspect Biol. 5,7(1): a020412a020412.

3. Li F, Geng X, Yip J, Ding Y (2019) Therapeutic Target and Cell-signal Communication of Chlorpromazine and Promethazine in Attenuating Blood-Brain Barrier Disruption after Ischemic Stroke. Cell Transplant. 28(2):145-156.

4. Engelhardt S, Patkar S, Ogunshola 00 (2014) Cell-specific blood-brain barrier regulation in health and disease: a focus on hypoxia. Br J Pharmacol. 171(5):1210-1230

5. Abbott NJ, Patabendige AAK, Dolman DEM, Yusof SR, Begley DJ (2010) Structure and function of the blood-brain barrier. Neurobiol Dis. 37(1):13-25 
6. Huang S-F, Fischer S, Koshkin A, Laczko E, Fischer D, Ogunshola OO (2020) Cell-specific metabolomic responses to injury: novel insights into blood-brain barrier modulation. Sci Rep. 10(1):7760. https://doi.org/10.1038/s41598-020-64722-w

7. Haseloff RF, Krause E, Bigl M, Mikoteit K, Stanimirovic D, Blasig IE (2006) Differential protein expression in brain capillary endothelial cells induced by hypoxia and posthypoxic reoxygenation. Proteomics. 6(6):1803-1809

8. Li L, Welser-Alves J, van der Flier A, Boroujerdi A, Hynes RO, Milner R (2012) An angiogenic role for the a5 $\beta 1$ integrin in promoting endothelial cell proliferation during cerebral hypoxia. Exp Neurol. 237(1):46-54

9. Hellsten J, West MJ, Arvidsson A, Ekstrand J, Jansson L, Wennström M, et al (2005) Electroconvulsive seizures induce angiogenesis in adult rat hippocampus. Biol Psychiatry. 58(11):871-878

10. Betz AL, Firth JA, Goldstein GW (1980) Polarity of the blood-brain barrier: distribution of enzymes between the luminal and antiluminal membranes of brain capillary endothelial cells. Brain Res. 192(1):17-28

11. McConnell HL, Kersch CN, Woltjer RL, Neuwelt EA (2017) The Translational Significance of the Neurovascular Unit. J Biol Chem. 292(3):762-770

12. Elmorsy E, Al-Ghafari A, Almutairi FM, Aggour AM, Carter WG (2017) Antidepressants are cytotoxic to rat primary blood brain barrier endothelial cells at high therapeutic concentrations. Toxicol In Vitro. 44:154-163

13. Elmorsy E, Elzalabany LM, Elsheikha HM, Smith PA (2014) Adverse effects of antipsychotics on micro-vascular endothelial cells of the human blood-brain barrier. Brain Res. 1583(1):255-268 http://dx.doi.org/10.1016/j.brainres.2014.08.011

14. Chen C, Gonzalez FJ, Idle JR (2007) LC-MS-based metabolomics in drug metabolism. Drug Metab Rev. 39(2-3):581-597

15. Artati A, Prehn C, Adamski J (2019) LC-MS/MS-Based Metabolomics for Cell Cultures. Methods Mol Biol. 1994:119-130

16. Fragoso-Vázquez MJ, Méndez-Luna D, Rosales-Hernández MC, Luna-Palencia GR, Estrada-Pérez A, Fromager B, et al (2021) Molecular modeling and LC-MS-based metabolomics of a glutaminevalproic acid (GIn-VPA) derivative on HeLa cells. Mol Divers. 25(2):1077-1089

17. Feingold KR (2000) Introduction to Lipids and Lipoproteins. In: Feingold KR, Anawalt B, Boyce A, Chrousos G, de Herder WW, Dhatariya K, et al., editors. South Dartmouth (MA)

18. Lipp N-F, Ikhlef S, Milanini J, Drin G (2020) Lipid Exchangers: Cellular Functions and Mechanistic Links With Phosphoinositide Metabolism. Front cell Dev Biol. 8:663

19. Cikánková T, Fišar Z, Bakhouche Y, L'upták M, Hroudová J (2019) In vitro effects of antipsychotics on mitochondrial respiration. Naunyn Schmiedebergs Arch Pharmacol. 392(10):1209-1223

20. del Campo A, Bustos C, Mascayano C, Acuña-Castillo C, Troncoso R, Rojo LE (2018) Metabolic Syndrome and Antipsychotics: The Role of Mitochondrial Fission/Fusion Imbalance. Frontiers in 
Endocrinology. 9:144

21. Scaini G, Quevedo J, Velligan D, Roberts DL, Raventos H, Walss-Bass C (2018) Second generation antipsychotic-induced mitochondrial alterations: Implications for increased risk of metabolic syndrome in patients with schizophrenia. Eur Neuropsychopharmacol. 28(3):369-380

22. Elmorsy E, Alelwani W, Kattan S, Babteen N, Alnajeebi A, Ghulam J, et al (2021) Antipsychotics inhibit the mitochondrial bioenergetics of pancreatic beta cells isolated from CD1 mice. Basic Clin Pharmacol Toxicol. 128(1):154-168. https://doi.org/10.1111/bcpt.13484

23. Qing H, Xu H, Wei Z, Gibson K, Li XM (2003) The ability of atypical antipsychotic drugs vs. haloperidol to protect PC12 cells against MPP+-induced apoptosis. Eur J Neurosci. 17(8):1563-1570

24. Bonelli RM, Hofmann P, Aschoff A, Niederwieser G, Heuberger C, Jirikowski G, et al (2005) The influence of psychotropic drugs on cerebral cell death: female neurovulnerability to antipsychotics. Int Clin Psychopharmacol. 20(3):145-149

25. Li P, Wang Y, Liu X, Zhou Z, Wang J, Zhou H, et al (2019) Atypical antipsychotics induce human osteoblasts apoptosis via Wnt/ $\beta$-catenin signaling. BMC Pharmacol Toxicol. 20(1):10. https://doi.org/10.1186/s40360-019-0287-9

26. Tóth F, Cseh EK, Vécsei L (2021) Natural Molecules and Neuroprotection: Kynurenic Acid, Pantethine and a-Lipoic Acid. Int J Mol Sci. 22(1)

27. Weil-Malherbe H (1944) Metabolism of Acetoacetic Acid. Nature. 153(3884):435-436. https://doi.org/10.1038/153435c0

28. Stojanovic V, Ihle S (2011) Role of beta-hydroxybutyric acid in diabetic ketoacidosis: a review. Can Vet $\mathrm{J}=$ La Rev Vet Can. 52(4):426-430.

29. Bhatti JS, Bhatti GK, Reddy PH (2017) Mitochondrial dysfunction and oxidative stress in metabolic disorders - A step towards mitochondria based therapeutic strategies. Biochim Biophys Acta - Mol Basis Dis. 1863(5):1066-1077

30. Casares D, Escribá P V, Rosselló CA (2019) Membrane Lipid Composition: Effect on Membrane and Organelle Structure, Function and Compartmentalization and Therapeutic Avenues. Int J Mol Sci. 20(9)

31. Birben E, Sahiner UM, Sackesen C, Erzurum S, Kalayci O (2012) Oxidative stress and antioxidant defense. World Allergy Organ J. 5(1):9-19

32. Tan D-X, Manchester LC, Esteban-Zubero E, Zhou Z, Reiter RJ (2015) Melatonin as a Potent and Inducible Endogenous Antioxidant: Synthesis and Metabolism. Molecules. 20(10):18886-18906

33. Reiter RJ, Tan DX, Rosales-Corral S, Galano A, Zhou XJ, Xu B (2018) Mitochondria: Central Organelles for Melatonin's Antioxidant and Anti-Aging Actions. Molecules. 23(2)

34. Álvarez-Diduk R, Galano A, Tan DX, Reiter RJ (2015) N-Acetylserotonin and 6-Hydroxymelatonin against Oxidative Stress: Implications for the Overall Protection Exerted by Melatonin. J Phys Chem B. 119(27):8535-8543. https://doi.org/10.1021/acs.jpcb.5b04920 
35. Alvarez-Herrera S, Escamilla R, Medina-Contreras O, Saracco R, Flores Y, Hurtado-Alvarado G, et al (2020) Immunoendocrine Peripheral Effects Induced by Atypical Antipsychotics. Front Endocrinol (Lausanne). 11:195

36. Otręba M, Zdybel M, Pilawa B, Beberok A, Wrześniok D, Rok J, et al (2015) EPR spectroscopy of chlorpromazine-induced free radical formation in normal human melanocytes. Eur Biophys $\mathrm{J}$. 44(5):359-365

\section{Tables}

Due to technical limitations, tables are only available as a download in the Supplemental Files section.

\section{Figures}

RT: $0.00-15.50$

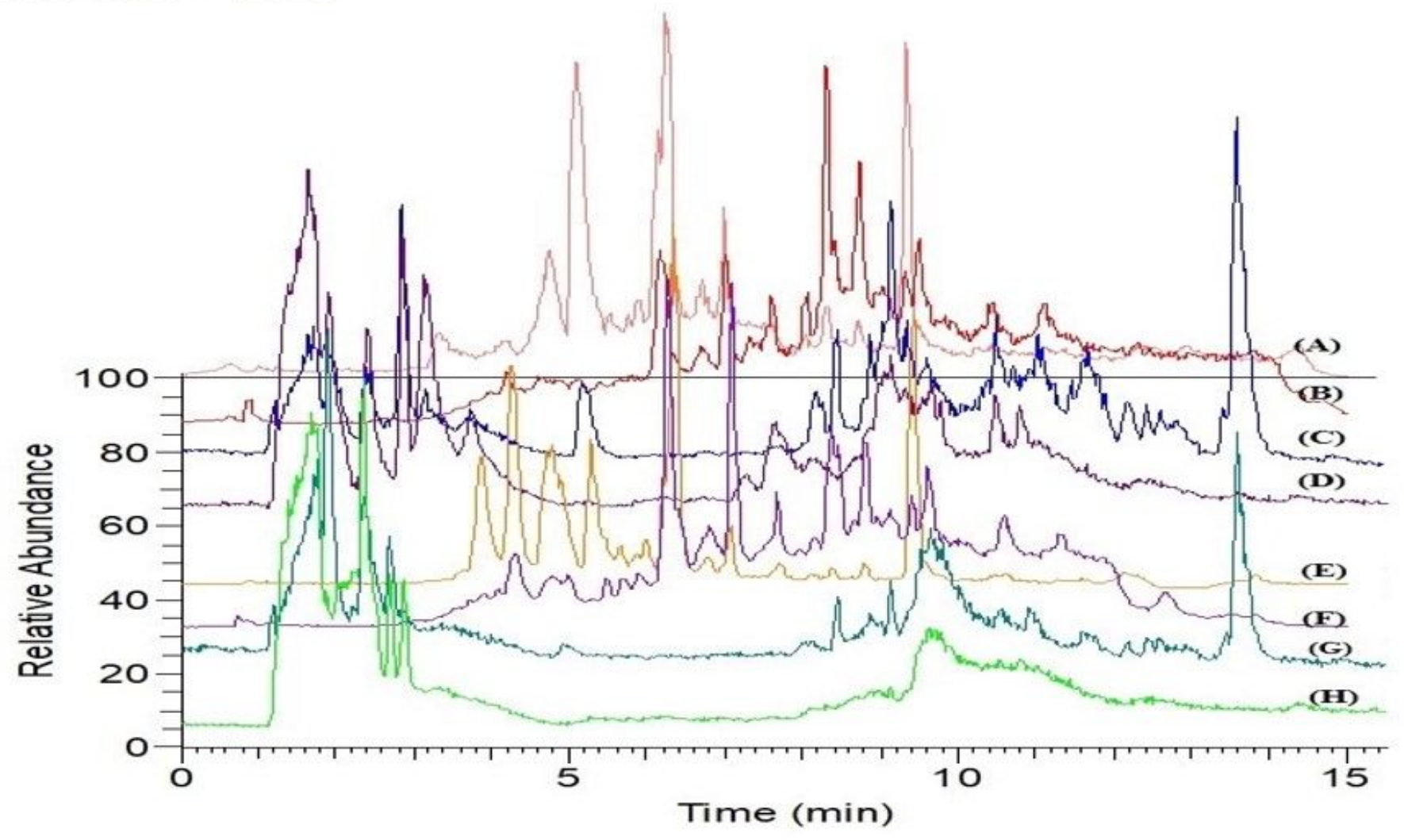

\section{Figure 1}

Total ion chromatogram (TIC) of non-polar fraction [in ESI+ (a) and ESI- (b)], polar fraction [in ESI+ (c) and ESI- (d)] of chlorpromazine-treated brain ECs. TIC of pooled QC samples in non-polar fraction [in ESI+ (e) and ESI-(f)] and polar fraction [in ESI+(g) and ESI-(h)]. 

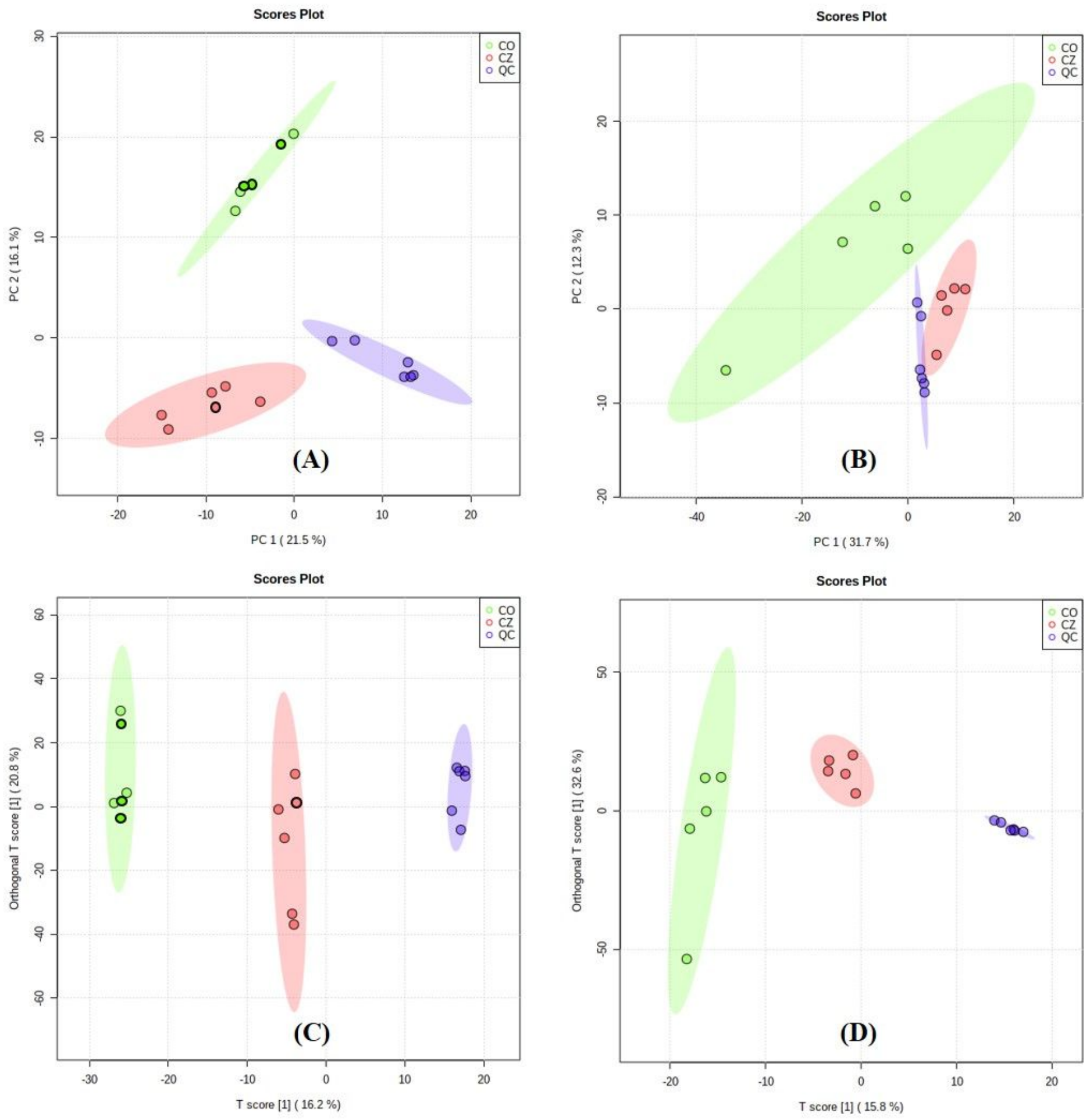

Figure 2

PCA scores plots of non-polar fraction in ESI+ (A) and in ESI- (B), and OPLS-DA scores plots of non-polar fraction in ESI+ (C) and in ESI- (D) of brain ECs treated with chlorpromazine. 
Dimethyluric acid

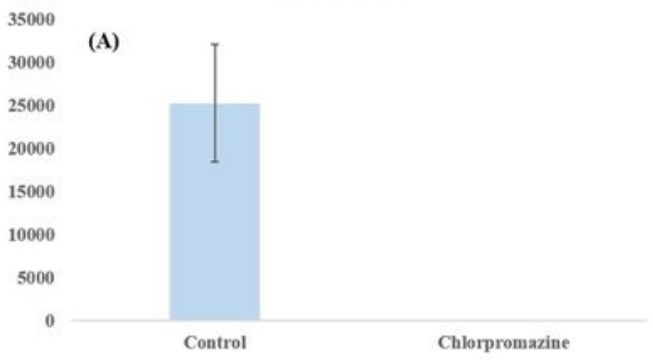

$8 Z, 11 Z, 14 Z$-eicosatrienoyl-CoA

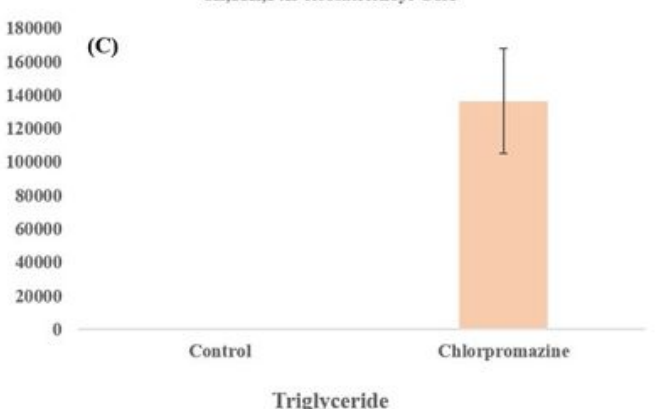

4500000

4000000

3500000

300000

2500000

2000000

1500000

1000000

500000

0

(E)

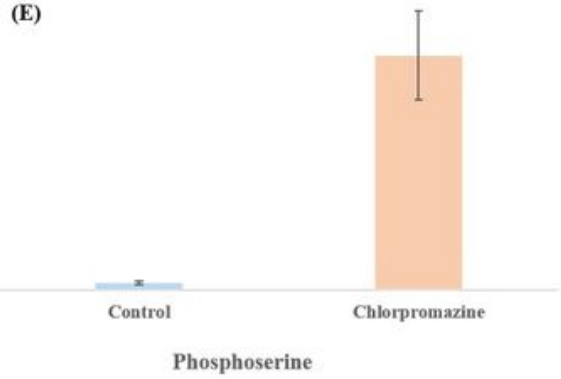

(G)

25000000

200000

15000000

10000000

5000000$$
0
$$
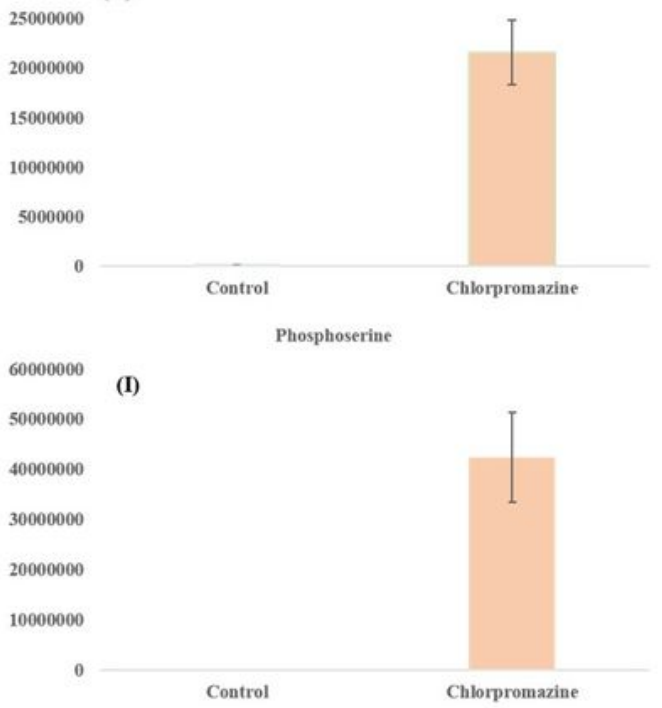

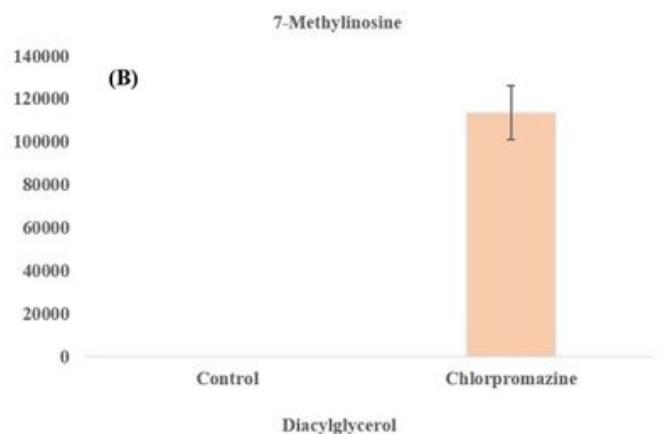

1600000

1400000

1200000

1000000

800000

600000

400000

200000

o

(D)

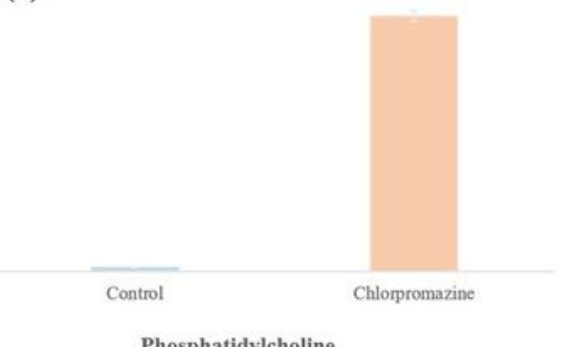

40000000

35000000

30000000

25000000

20000000

15000000

10000000

5000000

o

(F)

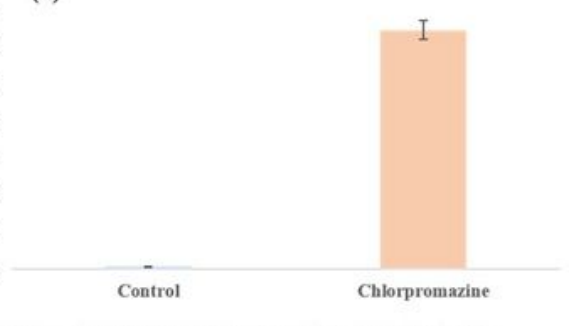

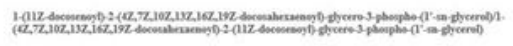

(H)

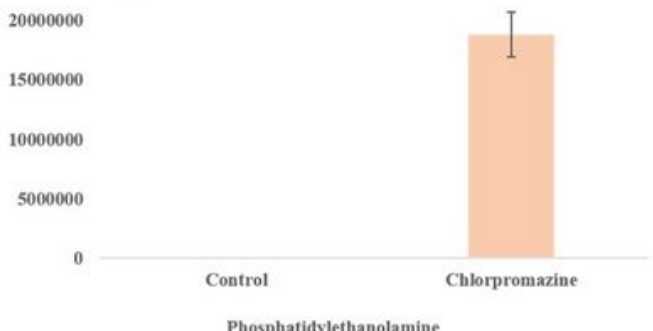

20000000

18000000

16000000

14000000

12000000

10000000

8000000

6000000

4000000

2000000

0
(J)

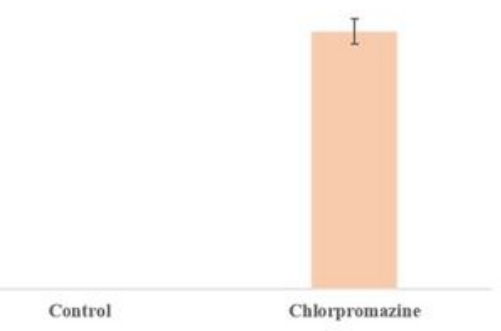

\section{Figure 3}

Level changes of the top ten significantly-changed metabolites from the non-polar fraction in brain ECs treated with chlorpromazine. 

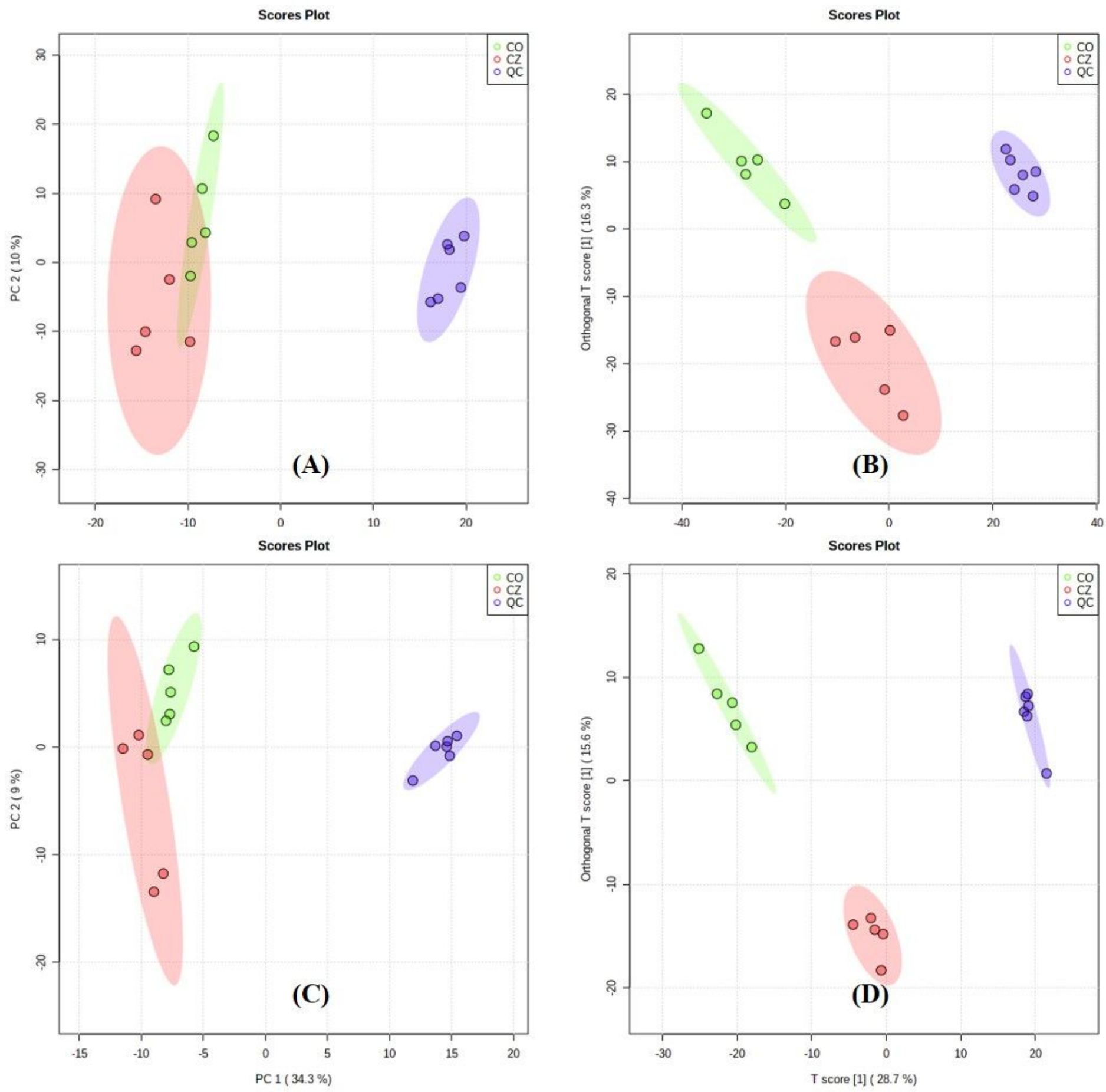

Figure 4

PCA scores plots of polar fraction in ESI+ (A) and in ESI- (B), and OPLS-DA scores plots of polar fraction in ESI+ (C) and in ESI- (D) of brain ECs treated with chlorpromazine. 

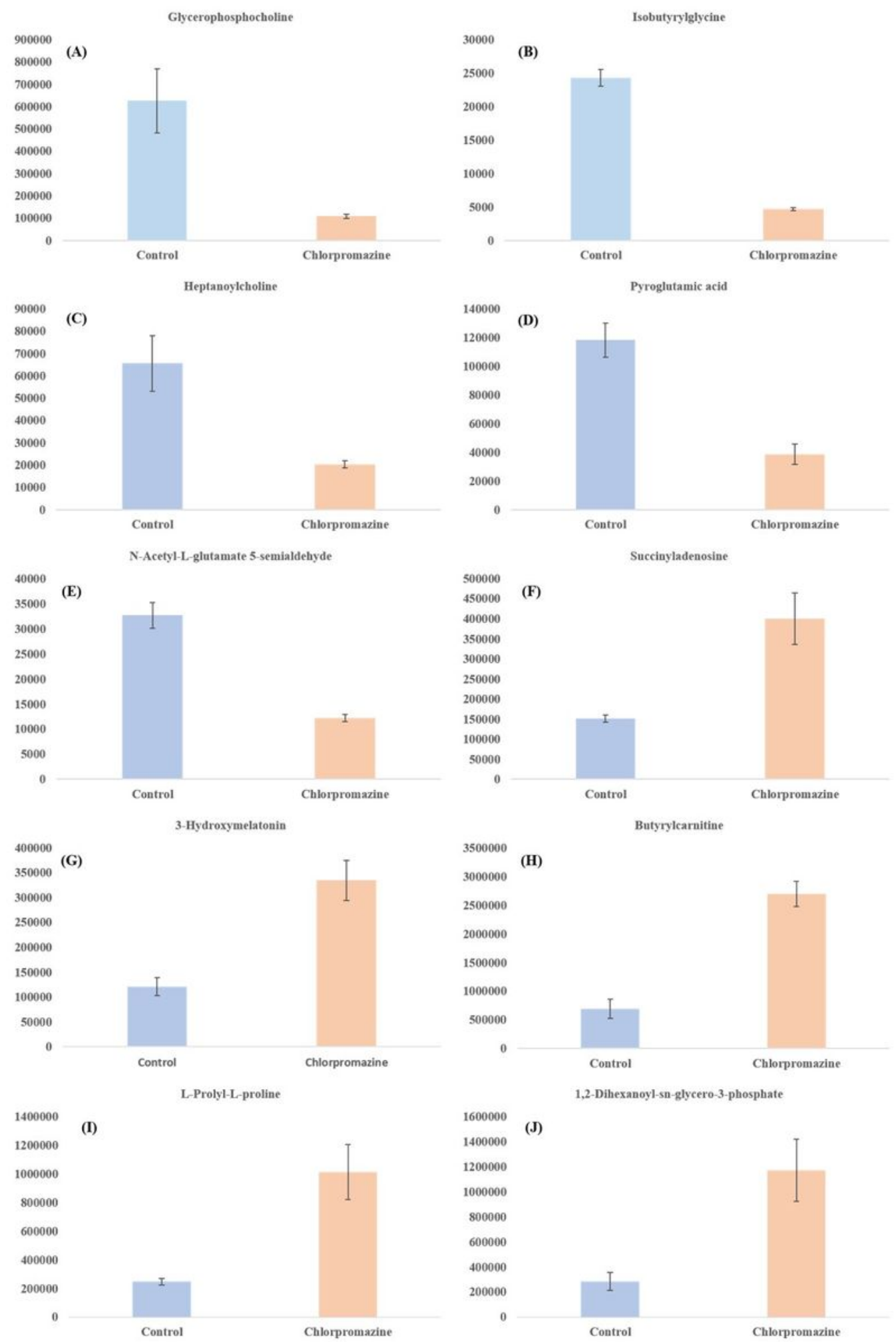

\section{Figure 5}

Level changes of the top ten significantly-changed metabolites from the polar fraction in brain ECs treated with chlorpromazine. 


\section{3-hydroxyactanoyl carnitine}

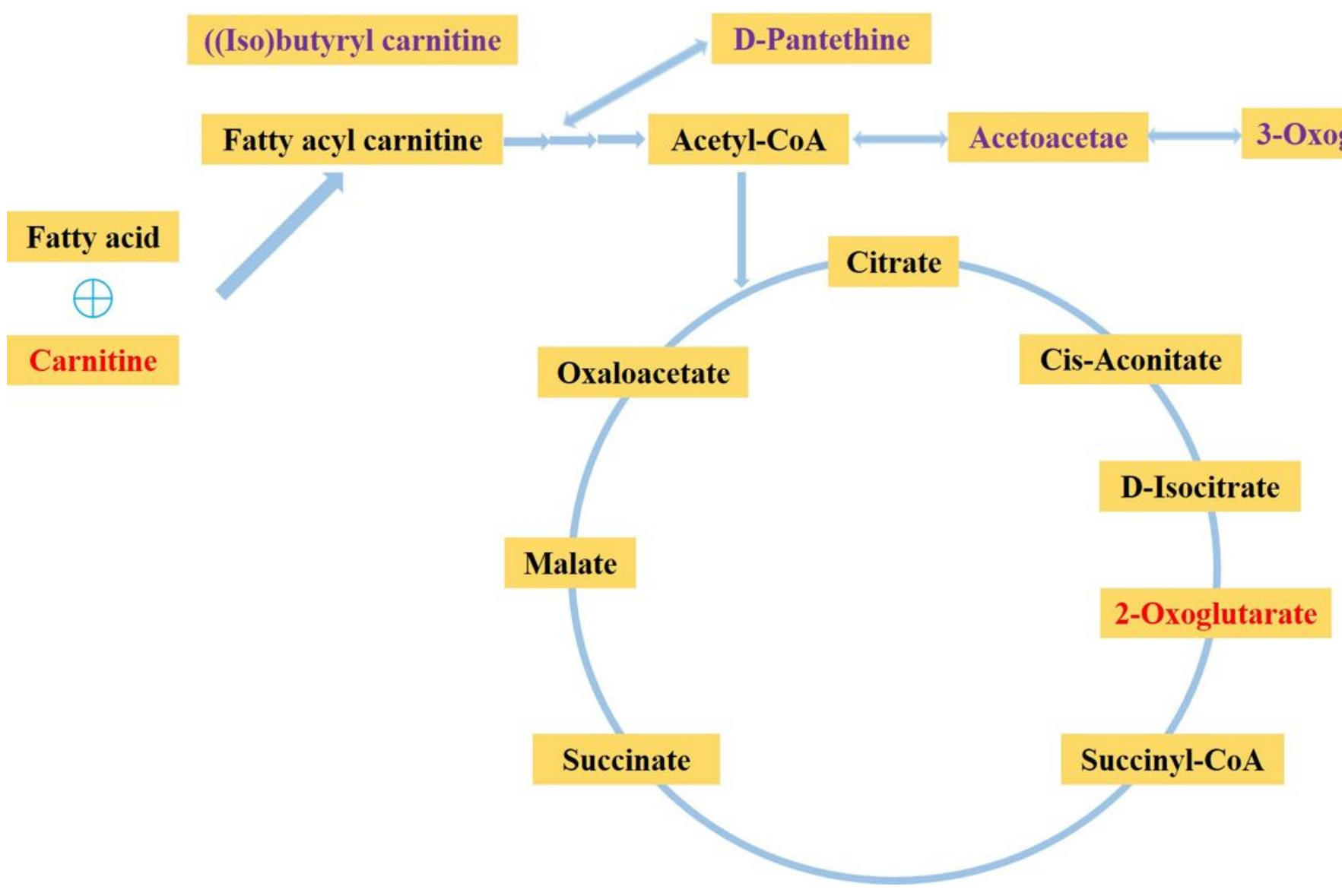

Figure 6

Overall simple diagram for biological pathway that is affected by high therapeutic dosage of chlorpromazine in brain ECs. Significantly changed metabolites are marked in red (decrease) and violet (increase).

\section{Supplementary Files}

This is a list of supplementary files associated with this preprint. Click to download.

- Table1.pdf

- Table2.pdf

- SupplementaryTable1.Significantlyalteredmetabolitesinnonpolarfraction.docx

- SupplementaryTable2.Significantlyalteredmetabolitesinpolarfraction.docx 